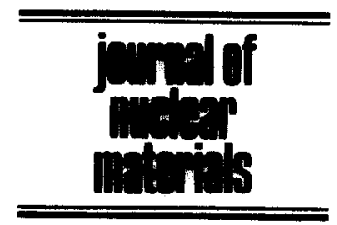

\title{
Intercomparison of microchemical evolution under various types of particle irradiation
}

\author{
G.S. Was and T. Allen \\ Department of Nuclear Engineering, University of Michigan, Ann Arbor, MI 48109, USA
}

\begin{abstract}
The problem of irradiation-assisted stress corrosion cracking (IASCC) in reactor cores is currently being addressed using different types of particle irradiation (electrons, heavy and light ions) to study the effect of neutron damage. Differences among the types of irradiation include particle type, temperature, dose and dose rate. In using different types of particle irradiation, the issue becomes how to determine whether a particular irradiation experiment produces the same effect as neutron irradiation in terms of a measurable quantity such as the amount of grain boundary depletion of chromium in 304 (Fe-20Cr-9Fe) stainless steel. Results of computer modeling experiments indicate that the expected amount of segregation is a strong function of displacement efficiency (particle type) and dose rate at constant dose. Charged particle irradiation has a higher efficiency than neutron irradiation and is generally conducted at a higher displacement rate requiring larger doses to achieve the same measure of radiation effect. The expected amount of segregation is also a sensitive function of the proximity to steady state.
\end{abstract}

\section{Introduction}

In the 1960 s and 70 s, heavy ion irradiation was used for the purpose of simulating neutron damage in support of the fast breeder reactor program. Ion irradiation has also been used to simulate the effects of 14 $\mathrm{MeV}$ neutron damage and simultaneous $\mathrm{He}$ injection in conjunction with the fusion reactor engineering program. Lately, another application of ion irradiation to the study of neutron irradiation damage has come to the forefront of the reactor community - that of irradiation-assisted stress corrosion cracking (IASCC). IASCC is the acceleration of the stress corrosion cracking process by irradiation. In a practical sense, the problem is primarily associated with the accelerated intergranular cracking process in austenitic alloys in light-water reactor (LWR) cores by neutron irradiation. Neutron irradiation has been found to cause a rise in the intergranular cracking and a decrease in ductility above $\sim 5 \times 10^{20} \mathrm{n} / \mathrm{cm}^{2}(E>1 \mathrm{MeV})$ [1]. The appearance of a "threshold" demonstrates that the observed behavior is due to irradiation-induced changes in the alloy that may involve microstructural changes, microchemical changes, compositional changes by transmutation, and synergism between these changes.

A problem has arisen concerning the comparison of results from neutron and charged particle irradiation experiments. How, for example is one to compare the results of a component irradiated in core at $288^{\circ} \mathrm{C}$ to a fluence of $1 \times 10^{21} \mathrm{n} / \mathrm{cm}^{2}(E>1 \mathrm{MeV})$ over a period of 8.5 months, with an ion irradiation experiment using $5 \mathrm{MeV} \mathrm{Ni}^{2+}$ at $500^{\circ} \mathrm{C}$ to $10 \mathrm{dpa}$ (displacements per atom) at a dose rate of $10^{-3} \mathrm{dpa} / \mathrm{s}$ ? The first question to resolve is the measure of radiation effect. In the IASCC problem, concern has centered on the effect of radiation on inducing the segregation of deleterious species to grain boundaries which then cause embrittlement or enhance the intergranular stress corrosion cracking (IGSCC) process. The appropriate measure of the radiation effect in this case would then be the impurity concentration at the grain boundary or the amount of impurity segregated to the grain boundary. This is a measurable quantity, either by Auger electron spectrometry (AES) or energy dispersive spectroscopy via scanning transmission electron microscopy (STEMEDS). Hence, a specific and measurable effect of radiation can be determined for both neutron and ion irradiation experiments.

The next concern is determining how ion irradiation translates into the environment describing neutron irradiation. That is, what are the irradiation conditions required for ion irradiation to yield the same measure of radiation effect as that for neutron irradiation? This is the key question, for in a postirradiation test program, it is only the final state of the material which is 
important in the determination of equivalence, and not the path taken. As such, one could probe (with a given ion) the temperature, dose and dose rate space until a coordinate is identified which produces the same measure of radiation effect as neutron irradiation. However, we are interested in a more orderly approach to the problem. To determine a priori the conditions of the ion experiment needed to produce the same measure of radiation effect as neutron irradiation, we must be able to account for the differences between the two experiments. These differences are four fold: particle type, temperature, dose and dose rate. The first of these differences presents its own unique set of problems, while the remaining differences can be resolved in the temporal description of the radiation damage process. It is the objective of this paper to compare the development of the microchemistry for different particle irradiations, and secondly, to outline a general method for approaching the problem of microstructural equivalence between ion and neutron irradiation to enable the use of ion irradiation in the solution of the IASCC problem.

\section{Methodology}

The first problem in determining the equivalence between the measure of radiation effect in charged particle and neutron irradiation is the use of a common dose unit. The basic (measurable) dose unit for neutron irradiation is $\mathrm{n} / \mathrm{cm}^{2}(E>x \mathrm{MeV})$ where $x$ is some energy threshold. For charged particles it is the integrated current or charge, $Q / \mathrm{cm}^{2}$. The particle beam community is accustomed to reporting dose in units of dpa and dose rate as dpa/s using one of several models for the determination of dpa. Although somewhat more complicated, due to the existence of an energy spectrum rather than a monoenergetic ion beam, the same conversion can be made from $\mathrm{n} / \mathrm{cm}^{2}$ $(E>x \mathrm{MeV})$, although it is seldom done. Nevertheless, the "ballistically" determined valuc of dpa calculated using a variant of the Kinchen-Pease displacement model [2] is not the appropriate unit to be used for dose comparisons. The reason is the difference in the primary damage state among different particle types.

Fig. 1 shows the relationship between damage morphology (as quantified by the average energy transfer in a PKA collision) and the displacement efficiency for various particle types impinging on a sample of pure nickel at $1 \mathrm{MeV}$. The displacement efficiency is defined as the fraction of the "ballistically" produced
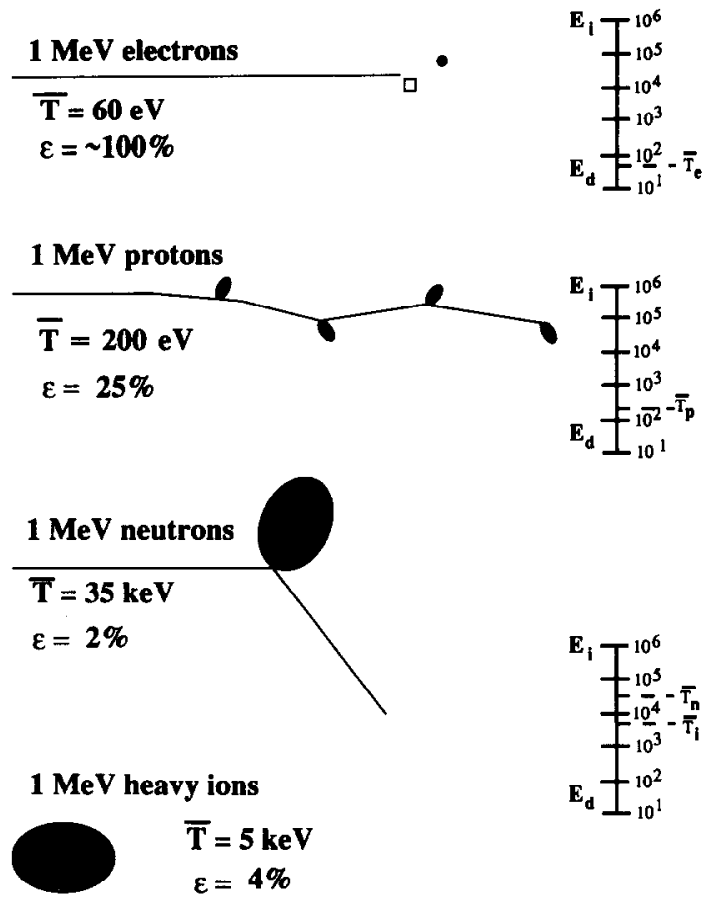

Fig. 1. Description of damage morphologies for irradiation with various particles of the same energy. $T$ is the average energy transfer per PKA and $\epsilon$ is the efficiency of producing freely migrating defects available to affect radiation-induced segregation.

Frenkel pairs (FPs) which survive the cascade quench and are available for long range migration. These are referred to variously as "freely migrating" [3] or "available migrating"' [4] defects. They are the only defects which will affect the amount of grain boundary segregation, our measure of radiation effect. The fraction of the total number of defects produced which are "freely migrating" is termed the displacement efficiency, $\epsilon$. Despite the equivalence in energy among the four particle types, the average energy transferred and the defect production efficiencies vary y almost two orders of magnitude!. This is explained by the differences in the cascade morphology among the different particle types. Neutrons and heavy ions produce dense cascades which result in substantial recombination during the cooling or quenching phase. However, electrons are just capable of producing a few widely spaced Frenkel pairs (FP) which have a low probability of recombination. Protons produce small widely spaced cascades and many isolated FPs due to the Coulomb interaction and therefore, fall between the extremes in 
displacement efficiency defined by electrons and neutrons.

We will focus on the comparison between four types of particle irradiation to outline a methodology for establishing equivalence between neutron and charged particle irradiation. The four types are given in table 1 and are taken from experiments conducted to study the IASCC problem [5-8]. Each experiment is characterized by the particle type and energy, irradiation temperature, reported dose rate and reported total dose. The displacement efficiency is calculated using Naundorf's model [9] which is based on two factors. The first is that energy transfer to atoms is only sufficient to create a single Frenkel pair. The second is that the Frenkel pair lie outside a recombination (interaction) radius so that the nearby FPs neither recombine nor cluster. The model follows each generation of the collision and calculates the fraction of all defects produced that remain free. Results of the model are the displacement efficiencies shown for the four particle types in table 1. The "real" displacement rate and "real" total dose for each particle type are determined by multiplying the reported (uncorrected) values times the efficiency factor.

As a means of comparing the expected measured amount of grain boundary segregation for each of the irradiations described in table 1, we used the Lam model [10] to simulate the depletion of chromium at the grain boundary of 304 stainless steel of composition $\mathrm{Fe}-20 \mathrm{Cr}-9 \mathrm{Ni}$. This model follows the theory of radiation-induced segregation in concentrated alloys and amounts to the solution of a system of four (two alloying elements, vacancies and interstitials) coupled partial differential equations describing the evolution of defect and atom distributions in time and space. The equation for the third alloying element is not needed because it is not independent, ie. $C_{\mathrm{C}}=1-C_{\mathrm{A}}-C_{\mathrm{B}}$. Numerical solutions are made for a sample under uniform irradiation with energetic particles assuming a spatially uniform production rate of defects for each particle type. Particle type is identified by the input displacement rate. Model calculations are made using the same assumptions, boundary conditions and material parameters as given by Lam et al. [10]. All cases assume an infinite medium with a planar sink. These conditions are strictly true for proton and neutron irradiations and were also shown to be valid for ion irradiation provided the experimental measurements are confined to the first $1 \mu \mathrm{m}$ of the sample where the displacement profile is flat. Since Kato used $400 \mathrm{~nm}$ thick TEM foils and most of the segregation is confined to within $20 \mathrm{~nm}$ of the grain boundaries, the foil

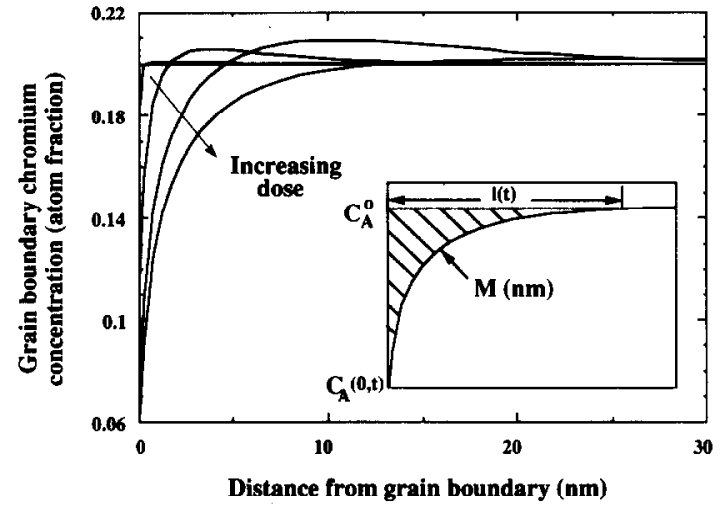

Fig. 2. Chromium concentration profile near the grain boundary in $\mathrm{Fe}-20 \mathrm{Cr}-9 \mathrm{Ni}$ during particle bombardment at a displacement rate of $10^{-3} \mathrm{dpa} / \mathrm{s}$ at $500^{\circ} \mathrm{C}$. The inset defines the variables used to describe the amount of $\mathrm{Cr}$ depletion, $M$, defined in the text.

thickness should not grossly affect the measured amount of segregation. Vacancies were assumed to migrate via the alloying elements with a migration energy of $1.3 \mathrm{eV}$, which is the same as the energy for vacancy migration in pure Fe. However, from the different values of $D_{0}$ measured experimentally [11], the preexponential attempt-frequency factors for $\mathrm{Ni}, \mathrm{Cr}$ and $\mathrm{Fe}$ were different. No preferential association of defects with $\mathrm{Fe}, \mathrm{Cr}$ or $\mathrm{Ni}$ atoms is assumed. Segregation of the alloying elements results from the difference in vacancy diffusion coefficients caused only by the inequality of the preexponential jump-frequency factors. There is no effect of interstitial fluxes because all the partial diffusion coefficients of interstitials via the three alloying elements are equal.

\section{Results and discussion}

Results show that enrichment of $\mathrm{Ni}$ and depletion of $\mathrm{Cr}$ and $\mathrm{Fe}$ occur in agreement with the calculations of Lam et al. [10,12]. The quantity of interest is the amount of chromium depleted from the grain boundary, or the area inside the $\mathrm{Cr}$ concentration profile, fig. 2. This quantity is measurable by STEM-EDS or by sputter depth profiling in AES. The amount of $\mathrm{Cr}$ depletion is determined by integrating the concentration profile for that element with distance $x$, from the grain boundary,

$M=\int_{0}^{l(t)}\left[C_{\mathrm{A}}^{0}-C_{\mathrm{A}}(x, t)\right] \mathrm{d} x$, 


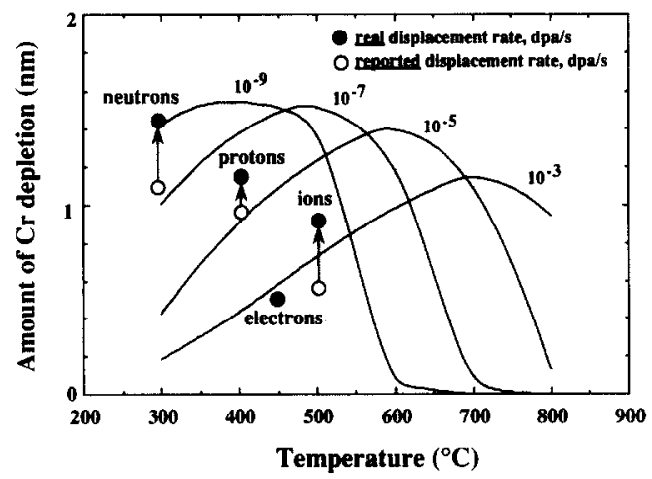

Fig. 3. Comparison of the amount of chromium depletion at steady state, as a function of temperature and dose rate, accounting for the displacement efficiency among the different particle types. The symbols represent the reported (uncorrected), and efficiency corrected dose rates for each particle type under the steady state conditions reported in table 1.

where $M$ is the segregated area, $C_{\mathrm{A}}^{0}$ is the bulk atom concentration, $C_{\mathrm{A}}(x, t)$ is the atom concentration near the surface, and $l(t)$ is the half-width of the depleted zone. The amount of $\mathrm{Cr}$ depletion for "real" and "reported" dose rates is given in table 1 for each experiment. Also given are the values of $\mathrm{Cr}$ depletion at steady state and the doses required to reach steady state. Fig. 3 shows the amount of $\mathrm{Cr}$ depletion as a function of temperature and displacement rate at steady state. Steady state is reached at different dose levels for each experiment. At a given displacement rate, the segregated area peaks at some intermediate temperature and falls off at both higher and lower temperatures. This is due to the dominance of recombination at low temperatures and back diffusion at high temperatures [13]. Also note that the effect of a decreasing displacement rate is to shift the curves to higher maxima at lower temperatures. For a given dose, a lower displacement rate yields lower steady state defect concentrations, reducing the number of defects lost to recombination, and shifting the curve to lower temperatures while increasing the degree of segregation.

Note the change in the calculated values for the amount of $\mathrm{Cr}$ depletion in the four experiments shown in fig. 3. Since electrons are assumed to be $100 \%$ efficient in producing defects available to affect segregation, there is no change in the segregated area after accounting for efficiency. However, there is a difference with protons, heavy ions and neutrons. The difference is largest for neutrons and smallest for protons. The difference is a function of not only the displace- ment efficiency, but also the slope of the dose rate curves. Nevertheless, substantial differences result in the expected amounts of grain boundary segregation when the displacement efficiency is taken into account.

Fig. 3 shows the effect of three of the four parameters defining an experiment: particle type, temperature and dose rate. It does not show the effect of dose since this is a steady state result which is achieved at different doses for each of the experiments described in table 1. Fig. 4 shows the dose required to reach steady state as function of temperature and dose rate. Each of the experiments are plotted for both the reported and the real displacement rates. Note the large difference in the dose to reach steady state between electrons and neutrons. In general, irradiation at a lower dpa rate will result in a lower dose at steady state and the difference is greatest for this comparison. Correspondingly, proton and heavy ion irradiation fall between neutrons and electrons for the experiments described in table 1.

The series of curves in figs. $5 \mathrm{a}$ and $5 \mathrm{~b}$ show the development of the segregated area due to chromium depletion (versus dose and irradiation time, respectively) during irradiation for each of the four experiments described in table 1. Each particle type generates a pair of curves, the top curve (at large dose) results from use of the real displacement rate and the bottom curve from use of the reported displacement rate. A single curve was used for electron irradiation since the efficiency of defect production for electrons was assumed to be $100 \%$. The point on the curves

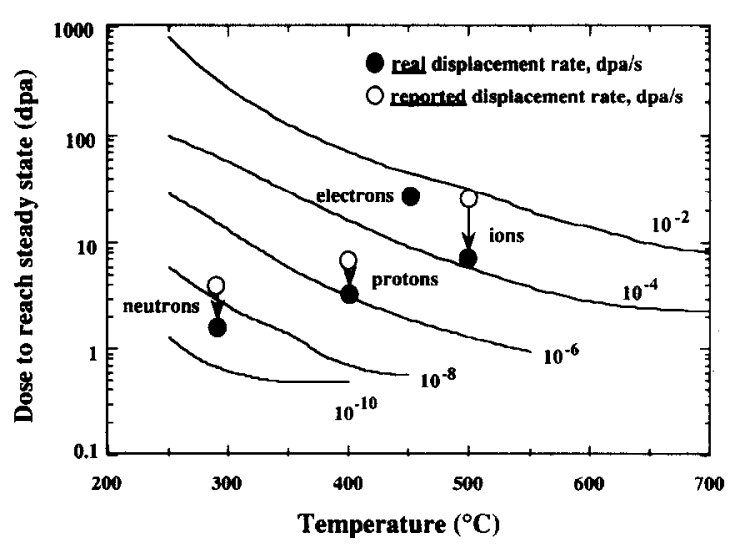

Fig. 4. Dose required to reach steady state as a function of temperature, dose rate and particle efficiency for each particle type. The symbols represent the reported (uncorrected), and efficiency corrected dose rates for each particle type under the steady state conditions reported in table 1. 


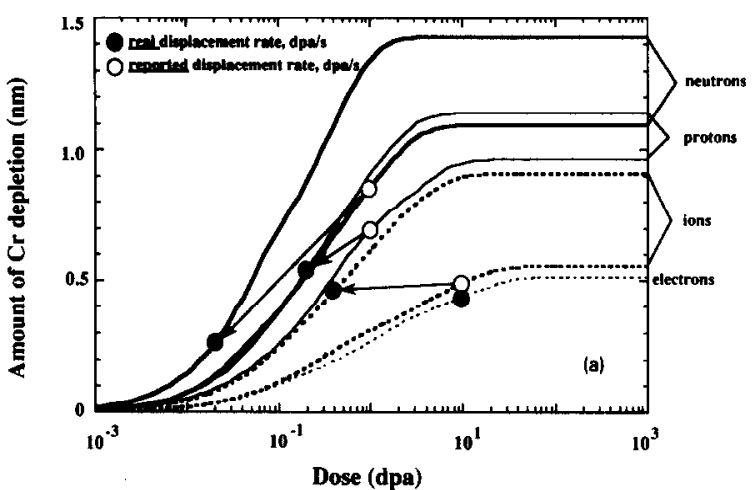

Fig. 5a. Amount of chromium depletion as a function of irradiation dose for each particle type. The upper curve (at steady state) in each pair accounts for the particle efficiency while the lower curve is for the reported (uncorrected) dose rate. The symbols refer to the conditions at the end of the experiments described in table 1.

indicate the doses (times) to which these experiments were conducted. The arrows indicate the changes in the measured depletion when the reported displacement rate and dose are corrected using the efficiency factors in table 1 . In none of the experiments is steady state reached after the reported dose. This is particularly true for electron irradiation where even after 10 dpa, the steady state dose is still a factor of 3 away. Even in the case of neutrons, a real dose of $1 \mathrm{dpa}$ (just past the threshold for observation of IASCC) falls on the steep portion of the curve, indicating that the

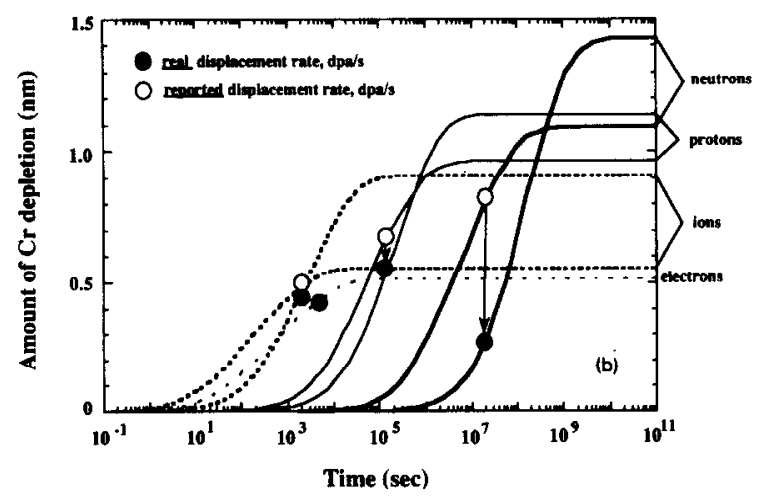

Fig. 5b. Amount of chromium depletion as a function of irradiation time for each particle type. The upper curve (at steady state) in each pair accounts for the particle efficiency while the lower curve is for the reported (uncorrected) dose rate. The symbols refer to the conditions at the end of the experiments described in table 1. measured amount of segregation is very sensitive to the duration of the experiment.

A final point of importance is the amount of $\mathrm{Cr}$ depletion that would be measured for each of the particle types after the stated duration of each experiment. Fig. 5a shows that for these four experiments, the amount can vary by almost a factor of 3 between various particle types. This is essentially an effect of the rate dependence on the amount of segregation. The curves in fig. 5a also show the interplay between rate and efficiency for a fixed amount of segregation. Low efficiencies or low displacement rates will produce greater amounts of segregation after a fixed dose than higher efficiencies or higher displacement rates. This trade-off works against experimenters using ion irradiation to study neutron damage. Generally, the efficiency is better for lighter ions, and due to the nature of the instrumentation (accelerators, HVEM microscope), damage rates are generally much higher.

Fig. 6 summarizes the resulting discrepancies between determination of the amount of segregation using the "reported" dpa rates and doses versus "real" values which account for the efficiency of producing freely migrating defects available for affecting segregation. As expected, the difference is generally largest for the particle type with the greatest correction to the dpa rate. However, the difference is strongly dependent on the dose to which the experiment is carried. In fact, ion irradiation experiments near $10 \mathrm{dpa}(0.4$ "real" dpa) will show virtually no discrepancy due to the cross-over of the segregation curves at that dose.

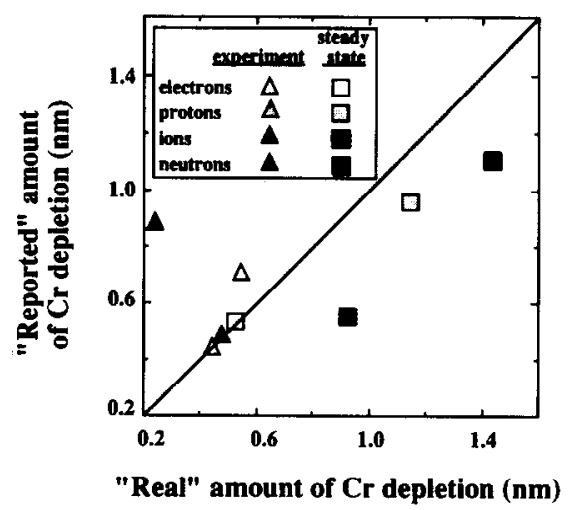

Fig. 6. Comparison of the amount of chromium depletion accounting for particle efficiency ("real" amount of Cr depletion) and that using the reported (uncorrected) values of dose and dose rate ("reported" amount of $\mathrm{Cr}$ depletion), for the duration of the experiment given in table 1 and at steady state. 
Returning to the example posed in the introduction, it is evident that ion irradiation with $5 \mathrm{MeV} \mathrm{Ni}^{2+}$ can be used to study neutron irradiation effects on grain boundary composition. According to table 1, the reported dose of $1 \times 10^{21} \mathrm{n} / \mathrm{cm}^{2}(E>1 \mathrm{MeV})$ corresponds to $\sim 1 \mathrm{dpa}$ or $0.02 \mathrm{dpa}$ corrected for efficiency. The curves of fig. $5 \mathrm{a}$ indicate that the amount of $\mathrm{Cr}$ depletion which can be expected under these conditions is $0.24 \mathrm{~nm}$. The same amount of depletion can be obtained using $5 \mathrm{MeV} \mathrm{Ni}^{2+}$ at $500^{\circ} \mathrm{C}$ at a dose rate of $5 \times 10^{-3} \mathrm{dpa} / \mathrm{s}\left(2 \times 10^{-4} \mathrm{dpa} / \mathrm{s}\right.$ efficiency corrected $)$ after about 0.1 real dpa (2.5 uncorrected dpa) or $500 \mathrm{~s}$ of irradiation. The same is true for proton irradiation to 0.05 "real" dpa or electron irradiation to 0.8 "real" dpa at the "real" displacement rates given in table 1). However, this only provides an equivalence on the basis of the total amount of segregation, and does not account for differences in the shape (depth and width) of the segregated profile. Obviously, the microstructure is not accounted for either.

This example also illustrates the methodology for determining equivalence between grain boundary composition for irradiation with different particle types. In practice, the method may be used either to determine the irradiation conditions which achieve equivalence a priori, or to determine the relative amounts of grain boundary segregation "expected" given the parameters defining the experiment.

\section{Summary}

The main points of the analysis can be summarized as follows. The efficiency of producing "freely migrating" defects which are available for affecting processes such as segregation can have a large impact on the resulting degree of segregation. Thus, calculations performed to account for the measured segregation due to irradiation must also account for displacement efficiency: However, displacement rate also strongly affects the degree of grain boundary segregation after a given dose. Experiments which use charged particles to study neutron damage generally have higher displacement efficiencies and are conducted at higher displacement rates, requiring larger doses to achieve the same measure of radiation effect. The dose to which experiments are conducted is also very important in comparing segregation from various particle types. Since most experiments do not reach steady state, the amount of segregation is a sensitive function of the total dose. Hence, comparisons between particle types can yield fortuitous agreement or disagreement depending on the length of the experiment. The methodology described herein can be used either to design an experiment a priori, for the purpose of achieving equivalence of the radiation effect, or to determine "correction factors" to apply to an experiment already conducted.

\section{Acknowledgements}

The authors gratefully acknowledge the assistance of Deborah Damcott (UM) and N. Lam (ANL) for their help in the execution and interpretation of the code describing RIS in ternary systems, the General Electric Company and the National Science Foundation for partial financial support of T. Allen.

\section{References}

[1] P.L. Andresen, F.P. Ford, S.M. Murphy and J.M. Perks, Proc. 4th Int. Symp. on Environmental Degradation of Materials in Nuclear Power Systems - Water Reactors, National Association of Corrosion Engineers, Houston, TX, 1990, pp. 1-83.

[2] G.H. Kinchin and R.S. Pease, Rep. Prog. Phys. 18 (1955) 1.

[3] L.E. Rehn, J. Nucl. Mater. 174 (1990) 144.

[4] L.K. Mansur and K. Farrell, J. Nucl. Mater. 170 (1990) 236.

[5] T. Kato, H. Takahashi and M. Izumiya, J. Nucl. Mater. 189 (1992) 167.

[6] D. Damcott, D. Carter, J. Cookson, J. Martin, M. Atzmon and G.S. Was, Radiat. Eff. Def. Solids 118 (1991) 383.

[7] S. Bruemmer, J. Nucl. Mater. 186 (1991) 13.

[8] A.J. Jacobs and G.P. Wozadlo, Corrosion 91, National Association of Corrosion Engineers, Houston, TX, 1991, paper \#41.

[9] V. Naundorf, J. Nucl. Mater. 182 (1991) 254.

[10] N.Q. Lam, A. Kumar and H. Wiedersich, Proc. 11th Conf. on Effects of Radiation on Materials, ASTM-STP 782, eds. H.R. Brager and J.S. Perrin (American Society for Testing and Materials, Philadelphia, 1982) p. 985.

[11] S.J. Rothman, L.J. Nowicki and G.E. Murch, J. Phys. F10 (1980) 383.

[12] N.Q. Lam, J. Nucl. Mater. 117 (1983) 106.

[13] P.R. Okamoto and L.E. Rehn, J. Nucl. Mater. 83 (1979) 2. 